\title{
Laser treatment in urogynaecology and the myth of the scientific evidence
}

\author{
G. Alessandro Digesu ${ }^{1,2} \mathbb{D} \cdot$ Steven Swift $^{3}$
}

Received: 2 July 2017 / Accepted: 10 August 2017 / Published online: 25 August 2017

(C) The International Urogynecological Association 2017

In 2015, we published an editorial in the International Urogynecology Journal on laser vaginal rejuvenation [1]. We questioned the literature and science behind the popular and trendy claims that laser vaginal treatment improved stress urinary incontinence and certain aspects of vaginal atrophy/sexual function, as they had not yet been substantiated by a significant body of evidence.

Despite these authors' suggestion that laser application as treatment for urogenital symptoms needs to be very cautiously appraised in view of the lack of a robust and qualitative body of data, we have seen little progress in the research direction. A quick check of clinicaltrials.gov reveals that several observational studies have been reported in the literature: one prospective double blinded trial comparing vaginal laser therapy with estriol cream that was completed in November 2015 (from Brazil) and one prospective double blinded trial comparing vaginal laser therapy with conjugated equine oestrogen cream, which is currently recruiting. There are several other safety and efficacy studies on the role of laser vaginal therapy as a treatment option for stress urinary incontinence (SUI), but no other comparative trials. The Brazilian study, which was completed in November 2015, was not found in the literature through a search using neither the topic nor the principal investigator. Nevertheless, the number of laser

G. Alessandro Digesu

a.digesu@imperial.ac.uk

1 Department of Urogynaecology, St Mary's Hospital, London, UK

2 Academic Department of Obstetrics and Gynaecology, Department of Urogynaecology, St Mary's Hospital, Mint Wing, South Wharf, London W2 1NY, UK

3 Department of Obstetrics and Gynaecology, Medical University of South Carolina, Charleston, SC, USA procedures for managing important aspects of vulvovaginal atrophy and/or "genitourinary syndrome of menopause (GSM) " and urinary incontinence in the last 3 years has increased significantly worldwide, mainly because of media advertising and manufacturer's marketing policy. To date, there have been approximately 15 laser companies on the market advertising laser vaginal treatment as an effective minimally invasive solution for a wide range of urogynecological disorders.

In this journal, in May 2017, Arunkalaivanan et al. conducted a well-designed systematic review of laser therapy as a treatment modality for the relief of symptoms of GSM [2]. Among a total 165 articles identified in their search, none was a randomised controlled trial (RCT) and none of the three observational studies included a control group. Additionally, the lack of RCTs made it difficult to undertake a meta-analysis, and thus to give any weight to the recommendations from the currently published studies. An important limitation of these short-term studies is that the potential risks of longterm complications, such as scarring, have not yet been addressed or reported. Therefore, assumptions cannot yet be made regarding the applicability or long-term effects of this treatment, whether positive or negative.

In this edition of the International Urogynecology Journal, Pergialiotis et al. have reported on the results of a systematic review on the role of laser vaginal therapy as a treatment option for SUI [3]. This review included 12 studies and a total of 760 patients who underwent laser therapy for SUI. No randomised controlled trials were identified. The authors found that the methodological quality of the reported studies was low, with Oxford Level Evidence 3b and 4. Several limitations including statistical bias, methodological flaws, the lack of a control group or comparison with other clinically proven treatment modalities (such as pelvic floor exercises, bulking agents or mid-urethral slings) were found, thus prohibiting firm conclusions regarding its use in the treatment of SUI. 
The conclusion was that although laser technology may hold promise for the future treatment of GSM, SUI and other urogynecological disorders, further robust, high-quality evidence from RCTs comparing laser with placebo or standard of care treatments, in addition to long-term efficacy and safety data, should be collected, before fully embracing this expensive new technology.

Despite this paucity of data, workshops and training courses are being promoted and offered at local, national and international scientific urogynecology meetings. This seems premature and the industry is driving the technology with very little scientific evidence to back any of the claims regarding benefits. It seems that we as conscientious investigators are losing control of a situation and are ignoring the potential risks associated with laser vaginal therapy in what we believe may become the next medicolegal situation in urogynaecology. This all sounds eerily similar to the lead-in of our current situation with transvaginal mesh. What role professional scientific societies have in guiding the development of a body of science behind a treatment modality, before offering that treatment to the general public, is still unknown. However, we now have experience in advising national agencies on what steps to take after a therapy that was promoted as safe and effective is deemed concerning and possibly unsafe. This was the role our professional societies filled as the concerns arose on an international level with trans-vaginal mesh for pelvic organ prolapse. This then becomes reactive damage control as opposed to a proactive responsible policy of guiding new promising technologies into clinical practice. Published guidelines for the review and/or selection process of submitted workshops' applications at annual international scientific meetings clearly state that the evaluation/selection criteria must include clinical relevance, scientific relevance and evidence-based merit. This means that the workshop content must be supported by evidence and must focus on high-quality research findings. In addition, it is clearly highlighted that the workshops should not promote a single product or procedure, as the subject matter should be generic, varied and unbiased. Are we adhering to these guidelines in the selection of workshops promoted at our local, national and international scientific meetings?

In conclusion, intravaginal laser therapy has become a very expensive and popular option among our peers for the treatment of urogynecological disorders, without any serious trials comparing active laser treatment with placebo or other standard of care therapies. It is performed on an outpatient or daysurgery basis and is mainly funded by the patient, with a cost of $\$ 600-1,500$ per visit, totalling $\$ 2,000-4,000$ per completed course of treatment. Unfortunately, 3 years later, we have to reiterate the same conclusions published in our previous editorial, that the therapeutic advantages of intravaginal laser treatment in urogynecology can only be recommended after robust clinical trials have demonstrated its safety and efficacy. Before those trials become available, it should only be offered in the setting of a clinical trial.

\section{Compliance with ethical standards}

Financial disclaimer/conflicts of interest GA Digesu: consultant (AMS, Astellas, Medtronic, Dompe, Standard Innovation Corporation), speaker honorarium (Allergan, Pfizer, Astellas, Uroplasty), trial participation (Allergan, Bluewind).

S Swift: research (Astellas, Cook)

\section{References}

1. Singh A, Swift S, Khullar V, Digesu GA. Laser vaginal rejuvenation: not ready for prime time. Int Urogynecol J. 2015;26(2):163-4. https://doi.org/10.1007/s00192-014-2588-2.

2. Arunkalaivanan A, Kaur H, Onuma O. Laser therapy as a treatment modality for genitourinary syndrome of menopause: a critical appraisal of evidence. Int Urogynecol J. 2017;28(5):681-5. https:// doi.org/10.1007/s00192-017-3282-y.

3. Pergialiotis V, Prodromidou A, Perrea DN, Doumouchtsis SK. A systematic review on vaginal laser therapy for treating stress urinary incontinence: do we have enough evidence? Int Urogynecol J. 2017. https://doi.org/10.1007/s00192-017-34. 